\title{
Mitochondrial uncoupling and the isodynamic equivalents of protein, fat and carbohydrate at the level of biochemical energy provision
}

\author{
BY GEOFFREY LIVESEY \\ AFRC Food Research Institute, Colney Lane, Norwich NR4 7UA
}

(Received 17 April 1984 - Accepted 29 October 1984)

1. The effects of uncoupling of mitochondrial oxidative phosphorylation on the efficiency of energy conservation during oxidation of amino acids, fatty acids, glycerol, glucose and 101 food proteins have been examined in order to compare how uncoupling at coupling site 1 affects energy yields compared with uncoupling at sites $2+3$ and uncoupling by proton leakage. The effects of uncoupling by each mechanism on the isodynamic equivalents of carbohydrate, fat and protein at the level of cytoplasmic ATP yield have been estimated.

2. Energy conservation during amino acid oxidation decreases relative to that for glucose as uncoupling by all three mechanisms increases. This effect is least when uncoupling is at site 1 and is associated with a fall in the isodynamic equivalent for protein : glucose of $4 \%$ maximally, and a fall in the cytoplasmic ATP yield for glucose of $25 \%$ (15-30\% when accounting for uncertainty in the choice of proton stoichiometries).

3. Variation in the efficiency of energy conservation for the different amino acids is large for both highly coupled and uncoupled mitochondria but the range of efficiencies for the oxidation of 101 food proteins is relatively small (less than $6 \%$ of the mean) for a tightly coupled system. This range increases absolutely (minimally fourfold) and relatively (minimally $44 \%$ of the mean value) with severely uncoupled mitochondria but is nearly constant (changes by less than $1 \%$ relative to the mean) within the probable physiologically relevant range of uncoupling in the whole body and in the full range of uncoupling at site 1 . The rank order position of particular proteins within the range of values is found to change most for gelatin which is oxidized with least energy conservation in a severely (unphysiologically) uncoupled system and most efficiently in a fully coupled system when oxidation of protein is considered to be direct, i.e. not via gluconeogenesis.

4. For medium- and long-chain fatty acids, uncoupling at site 1 elevates the efficiency of energy conservation relative to that for glucose (maximally $4 \%$ ) whereas uncoupling by other mechanisms decreases this relative efficiency. The pattern of effects for short-chain fatty acids resembles that for the amino acids.

5. The changes in the isodynamic equivalents of protein: glucose and of fat:glucose are small when uncoupling occurs at site 1 and tend to cancel for a mixed diet but are additive in the effect on food energy values when uncoupling is by the other mechanisms. Hence changes in the efficiency of oxidative energy coupling at site 1 in association with Luft's disease or dietary changes would result in effects which are of little true dietetic significance on the isodynamic equivalents of nutrients at the level of cytoplasmic ATP yield in vivo.

Metabolizable energy in foods is usually determined as the difference between gross energy intake and energy excreted, both measured by bomb calorimetry (Merril \& Watt, 1955; Miller \& Payne, 1959). Alternatively, the metabolizable energy in food can be estimated by the application of energy conversion factors, e.g. 4, 9, 4 and 3.75 for protein, fat, mixed carbohydrate and carbohydrate as monosaccharide respectively (Merril \& Watt, 1955; Paul \& Southgate, 1978) or by the use of regression equations relating the metabolizable energy to gross energy in foods and which also require information on the nitrogen in food and, in some instances, the dietary fibre or unavailable carbohydrate present (Miller \& Judd, 1984). All these methods express the metabolizable energy in joules or calories. Hill (1981) suggested that a more functional approach could be the expression of energy as ATP which is a universal currency of energy at the biochemical level. Although other biochemical energy currencies are known their contribution to energy expenditure is thought to be small and can, in any case, be equated to ATP. Whereas the metabolizable heat energy in food can be determined experimentally, the provision of ATP in the whole body from the oxidation of foodstuffs cannot be measured and so must be calculated on the basis of what is known about the metabolic pathways and the mechanisms by which the mitochondrion conserves 


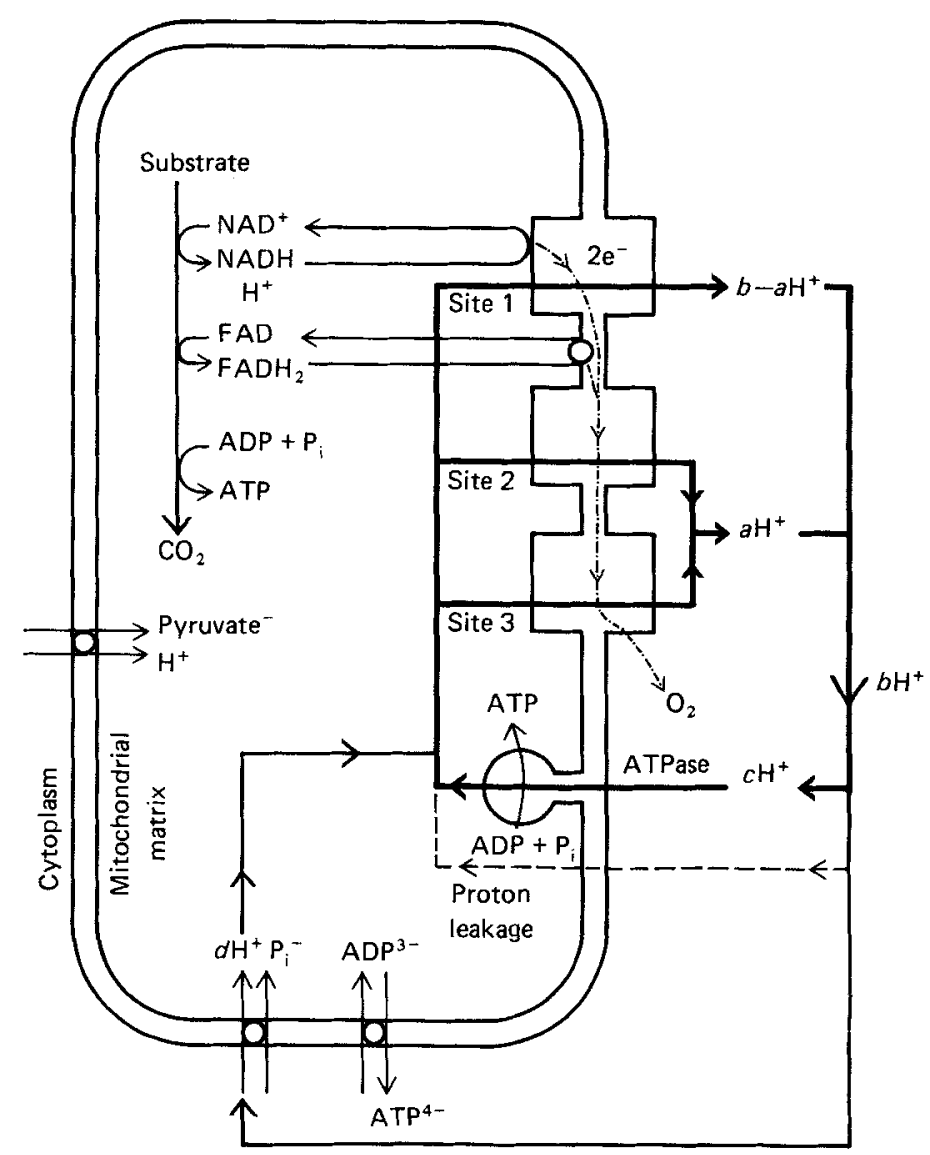

Fig. 1. Generation of cytoplasmic ATP during substrate oxidation. The mitochondrial respiratory chain contains three proton pumps (coupling sites 1,2 and 3 ). Reduced nucleotides (NADH, FADH ${ }_{2}$ ) generated at the level of substrate oxidation are re-oxidized giving rise to electrons which pass along the electron transfer chain (-..--) before being accepted by oxygen. The transfer of electrons results in the ejection of protons from the mitochondrial matrix into the cytoplasm. The numbers of protons ejected at each site remain a matter of debate (see Livesey, 1984) and are represented here by letters $a$ and $b$. Re-entry of a number of protons $(c)$ into the mitochondrial matrix through the ATP synthetase generates ATP within the mitochondrial matrix, whereas leakage of protons through the mitochondrial membrane at specific sites or through the normally proton-impermeable membrane results in no ATP synthesis. These proton movements constitute the proton circuit (Nicholls, 1982) and since each proton carries a net positive charge the proton movements are electrogenic. Non-electrogenic proton movements, e.g. pyruvate transport (-ve charge) with proton symport $(+v e$ charge) are electroneutral and do not affect the movement of charge along the proton circuit. The entry of inorganic phosphate $\left(\mathrm{P}_{\dot{1}}^{-}\right)$into the mitochondrial matrix for ATP synthesis occurs with proton symport and by itself is electroneutral. However, this occurs simultaneously with the transport of $\mathrm{ADP}^{3-}$ into the mitochondrial matrix with ATP ${ }^{4-}$ antiport so that in combination one proton and one net positive charge enter the matrix making an electrogenic proton movement which contributes to the proton circuit.

energy through oxidative phosphorylation. For tightly coupled systems (i.e. those which conserve energy as ATP maximally) such calculations indicate that the quantities of ATP generated per joule of carbohydrate or fat are similar, but that protein yields less ATP per metabolizable joule than either carbohydrate or fat (Krebs, 1964; Blaxter, 1967; Schulz, 1975; McGilvery, 1979; Livesey, 1984).

The events involved in the conservation of heat energy as ATP are illustrated in Fig. 1, 
which shows the transfer of electrons from the substrate to oxygen. The electron transfer is associated with the ejection of protons from the mitochondrial matrix into the cytoplasm which then re-enter the matrix through the ATP synthetase in support of ATP synthesis. Uncoupling of oxidative phosphorylation is possible either by disassociation of electron transfer from proton ejection or by disassociation of proton re-entry from ATP synthesis.

The precise efficiency with which mitochondria trap the heat energy in food or intermediary metabolites as ATP is not known, principally because of uncertainties in the proton stoichiometry of mitochondrial energy transduction $(a, b$, and $c$ in Fig. 1) and the extent to which mitochondrial oxidative phosphorylation is tightly coupled (Livesey, 1984). Nevertheless it is possible to show, within narrow limits, how changes in the efficiency of oxidative phosphorylation resulting from uncoupling affect the ATP energy available from protein, fat and carbohydrate when this is expressed relative to that for glucose (Livesey, 1984). It was shown that progressively increasing the extent of uncoupling of oxidative phosphorylation would progressively decrease the biochemical energy available from protein and fat relative to that available from glucose and that the extent of this effect is essentially independent of the types of uncoupling examined; that is proton leakage or short-circuiting (Nicholls, 1976, 1982) and the release of proton ejection from electron transfer at coupling sites $2+3$ (Skulachev, 1963; Chance, 1970; Ball, 1973). Further, this was essentially independent of the choice of proton stoichiometry ( $a, b$ and $c$ in Fig. 1) for tightly coupled mitochondria (Livesey, 1984). The overall effect of uncoupling at coupling site 1 on the isodynamic equivalents of the proximate constituents of foods at the biochemical level was not examined. While it is theoretically possible for site 1 to exhibit varied degrees of coupling, recent evidence indicates that in subjects with Luft's syndrome, a hypermetabolic mitochondrial myopathy associated with rapid fatigue (Morgan-Hughes, 1982), the efficiency of energy conservation at coupling site 1 is diminished (Morgan-Hughes et al. 1979; Clark et al. 1983). Further, in rats, dietary fat composition might influence the efficiency of energy conservation by selective uncoupling at site 1 (Deaver et al. 1984), although the extent to which this occurs in vivo has not been established.

The present paper shows that uncoupling at site 1 has effects on the isodynamic equivalents of the proximate constituents of foods that differ from those effects of uncoupling by either proton leakage or the release of proton ejection from electron transfer at sites $2+3$. At the level of cytoplasmic ATP yield even complete uncoupling at site 1 has effects which are of little true dietetic significance on the isodynamic equivalents of the proximate constituents of foods; this modifies a general statement on the effects of uncoupling made previously (Livesey, 1984).

\section{METHODS}

Heats of combustion of amino acids, fatty acids, glycerol and glucose; computation of the metabolizable heat energy of food proteins from food tables (Paul \& Southgate, 1978); computation of the net cytoplasmic ATP yields for amino acids, protein, fatty acids, glycerol and glucose, were all described in detail previously (Livesey, 1984) and are briefly described below together with appropriate modifications.

Metabolizable heat energy in each amino acid was calculated as the difference in its heat of combustion and the heat of combustion of the urea $(0.63 \mathrm{MJ} / \mathrm{mol})$ or ammonia $(0.32 \mathrm{MJ} / \mathrm{mol}$ ) (i.e. both $22 \mathrm{~kJ} / \mathrm{g} \mathrm{N}$ ) yielded on completion of catabolism. Metabolizable heat energy in protein was calculated as the sum of the metabolizable heat values for the constituent amino acids. For fatty acids, glycerol and glucose the metabolizable heat energy was taken as being equal to the corresponding heat of combustion.

To determine the effects of uncoupling on the cytoplasmic ATP equivalents of $\mathrm{FADH}_{2}$, 
mitochondrially generated NADH $\left(\mathrm{NADH}_{\text {mit }}\right)$, cytoplasmically generated NADH $\left(\mathrm{NADH}_{\mathrm{cyt}}\right)$ and ATP generated in the mitochondrial matrix at the substrate level $\left(\mathrm{ATP}_{\mathrm{mit}}\right)$, the following proton stoichiometries were used: $\mathrm{H}^{+}: \mathrm{O} \mathrm{NADH}$ mit $=9$ (b in Fig. 1), $\mathrm{H}^{+}: \mathrm{O}$ succinate $\left(\mathrm{FADH}_{2}\right)=6(a$ in Fig. 1$), \mathrm{H}^{+}:$ATP $F_{1}-F_{0}$ ATPase $=2(c$ in Fig. 1$)$ and $\mathrm{H}^{+}:$ATP transport $=1$ ( $d$ in Fig. 1). The cytoplasmic ATP equivalents of ATP generated in the cytoplasm $\left(\mathrm{ATP}_{\mathrm{cyt}}\right)$ is rigidly defined as equal to 1 . Substituting these values into eqns (1) to (4) permitted the calculation of the cytoplasmic ATP equivalents of $\mathrm{FADH}_{2}, \mathrm{NADH}_{\text {mit }}$, $\mathrm{NADH}_{\text {cyt }}$ and $\mathrm{ATP}_{\text {mit }}$ :

$$
\begin{gathered}
\text { FADH } \left._{2} \text { (succinate: } \operatorname{ATP}_{\text {cyt }}\right)=\left[a\left(1-U_{1}\right)\left(1-U_{2}\right)\right] /(c+d), \\
\mathrm{NADH}_{\text {mit }}=\left[(b-a)\left(1-U_{1}\right)\left(1-U_{3}\right)+a\left(1-U_{1}\right)\left(1-U_{2}\right)\right] /(c+d), \\
\mathrm{NADH}_{\mathrm{cyt}}=\mathrm{NADH}_{\mathrm{mit}}-[1 /(c+d)], \\
\operatorname{ATP}_{\text {mit }}=\mathrm{ATP}_{\mathrm{cyt}}-[1 /(c+d)],
\end{gathered}
$$

where $U_{1}, U_{2}$ and $U_{3}$ are the fractional uncoupling values for uncoupling by proton leakage $\left(U_{1}\right.$, which effectively modifies the values $a$ and $b$ ), uncoupling by dissociation of electron transfer from proton ejection at sites $2+3\left(U_{2}\right.$, which modifies the value of $\left.a\right)$ and dissociation of electron transfer from proton ejection at site $1\left(U_{3}\right.$, which modifies the value of $b-a$ ). Eqn (2) differs from the corresponding one in Livesey (1984) in order to account for uncoupling at site 1 (by introducting $U_{3}$ ). Eqn (3) is simplified from the corresponding equation in Livesey (1984) in order to account for uncoupling at site 1 and shows that the entry of an electrogenic proton (with a cytoplasmic ATP equivalent of $1 /(c+d)$ ) into the mitochondrial matrix for each transfer of a reducing equivalent from the cytoplasm to mitochondrial matrix (see Fig. 8.2 in Nicholls (1982)), decreases the cytoplasmic ATP equivalent of NADH that is generated cytoplasmically by comparison with mitochondrialmatrix generated NADH. The energy equivalent of this proton is not affected by uncoupling as implied in the corresponding equation published previously (p. 19 of Livesey, 1984). Transferring ATP generated at the substrate level from mitochondrial matrix to cytoplasm is also associated with the entry of an electrogenic proton into the mitochondrial matrix from the cytoplasm (Fig. 1). This decreases the energy equivalent of $\mathrm{ATP}_{\text {mit }}$ by $1 /(c+d)$ cytoplasmic ATP equivalents (eqn 4). With no uncoupling (by any mechanism) the fractional uncoupling values for $U_{1}, U_{2}$ and $U_{3}$ are each zero. Substituting these into eqns (1) to (4) therefore yielded cytoplasmic ATP equivalents for $\mathrm{FADH}_{2}=2$, $\mathrm{NADH}_{\text {mit }}=3, \mathrm{NADH}_{\mathrm{cyt}}=2.67$ and $\mathrm{ATP}_{\text {mit }}=0.67$ for the proton stoichiometries $a=6$, $b=9, c=2$ and $d=1$ (Fig. 1). In order to determine the effect of uncoupling on the cytoplasmic ATP equivalents of these 'high energy' intermediates, the following fractional uncouplng values were applied: $U_{1}=0.2,0.4,0.6$ and $0.8, U_{2}$ and $U_{3}=0.2,0.4,0.6,0.8$ and 1.0. The cytoplasmic ATP equivalents of each amino acid, fatty acid, glycerol and glucose under each condition of uncoupling were calculated from the number of each 'high-energy' intermediate generated in the corresponding oxidative pathway of metabolism and the appropriate cytoplsmic ATP equivalents of the 'high energy' intermediate as described in detail by Livesey (1984).

The available biochemical energies for amino acids, glycerol, fatty acids and glucose were calculated and expressed as the potential net cytoplasimic ATP yield in units of mol ATP/MJ metabolizable energy (Livesey, 1984). The available biochemical energy in protein was calculated as the sum of the cytoplasmic ATP yields for the constituent amino acids all divided by the sum of the metabolizable heat energy in the constituent amino acids.

Changes in the available biochemical energy were used as a measure of the change in the efficiency of energy conservation due to uncoupling. The isodynamic equivalence at the biochemical level was calculated as the glucose energy equivalent for amino acids, protein, fatty acids and glycerol by dividing the available biochemical energy for one substrate by 
the available biochemical energy for glucose (ratio, substrate: glucose energy in Figs. 2, 3 and 4). Whereas the metabolizable heat energy values assume complete absorption of nutrients from the gut (i.e. $100 \%$ digestibility), the available biochemical energy and substrate:glucose energy values each assume only that the absorbed and the corresponding faecal substrates have similar available biochemical energies (had the latter been absorbed).

\section{RESULTS AND DISCUSSION}

\section{Loss of the glucose energy}

While severely uncoupled mitochondria should be unable to support the energetic requirements of man, some indication of the maximum extent of uncoupling which might be tolerated can be obtained from observations of subjects with Luft's disease. Such individuals appear able to support an energy expenditure only a little above an already high resting metabolic rate, are rapidly fatigued and show loss of energy couplng at coupling site 1 in mitochondria in vitro (Morgan-Hughes et al. 1979; Morgan-Hughes, 1982; Clark et al. 1983). Complete loss of energy conservation at site 1 would result in the loss of approximately $25 \%$ of the available biochemical energy in glucose when compared with fully coupled mitochondria. When considering the uncertainty in the precise coupling ratios $(a$, $b$ and $c$ in Fig. 1), this loss may be as little as $15 \%$ or as large as $30 \%$ (values calculated on the basis of coupling ratios in columns $A$ and $F$, Table 1 , Livesey, 1984). These values represent potential losses only, since there is no evidence that mitochondia in vivo in subjects with Luft's disease exhibit the full extent of uncoupling at site 1 or that in normal subjects some energy loss by uncoupling is negligible.

\section{Isobioenergetic equivalents of nutrients}

The extent to which Rubner's (1902) concept of the isodynamic equivalents of nutrients remains valid at the level of net cytoplasmic ATP yield was questioned for instances when site 1 becomes selectively uncoupled. This is important since it was previously concluded (Livesey, 1984) that changes in the levels of energy coupling might require adjustment of the Atwater conversion factors. The effects of selective site 1 uncoupling are illustrated for amino acids (Fig. 2), food proteins (Fig. 3) and fatty acids (Fig. 4) with glucose being the standard reference (Livesey, 1984). Fat or fatty acid has been used as a reference standard (Blaxter, 1967) but this is not appropriate when considering food energy values because the heterogeneities in fat composition, heat energy value and efficiency of oxidation are greater than those for carbohydrate for which glucose is typical (Livesey, 1984). For comparison, the effects of uncoupling at sites $2+3$ and uncoupling by proton leakage are also shown. In Figs. $2-4$ the value 1.0 for the ratio, substrate: glucose energy, indicates that the number of moles of cytoplasmic ATP yielded per MJ of substrate is equal io the number of moles of cytoplasmic ATP yielded for each MJ of glucose oxidized.

The efficiency of energy conservation during amino acid oxidation differs from one amino acid to another and all differ from that for glucose (Fig. 2). The pattern of effects of uncoupling on this efficiency is, however, similar for each amino acid. Uncoupling by each mechanism results in greater losses of available biochemical energy from amino acids than from glucose. This effect is least for uncoupling at site 1 and, except for a small number of amino acids (glycine, serine, alanine, asparagine and arginine), the decrease is small. Fig. 2 was constructed on the basis that glucogenic amino acids are catabolized via glucose formation. A similar pattern of results was obtained for the direct oxidation of amino acids but with greater energy conservation for the glucogenic amino acids. This general pattern of results was also obtained for the 101 food proteins (Fig. 3). Here the range of energy 

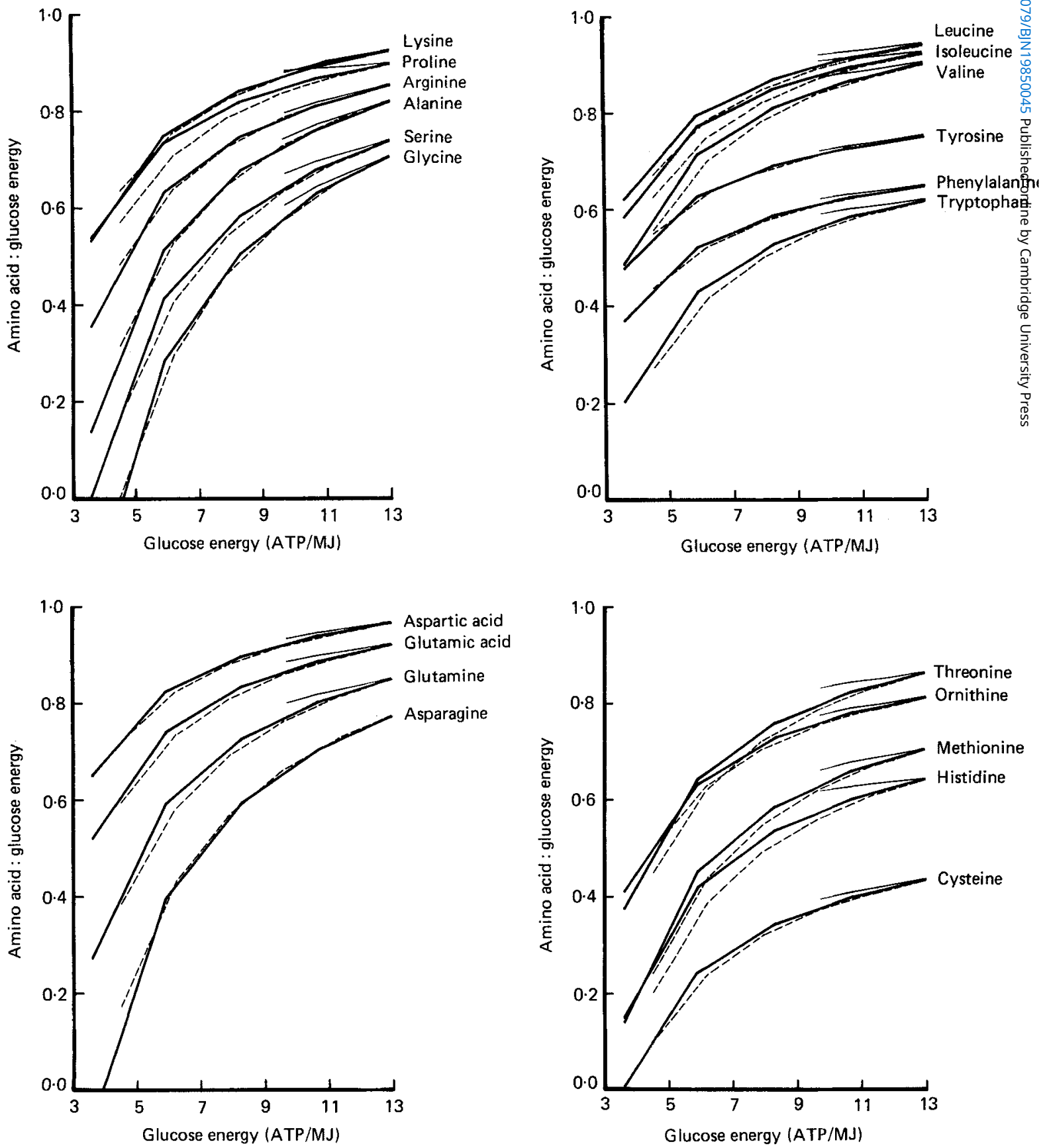

Fig. 2. Change in the efficiency of oxidation of amino acids relative to that for glucose. The decrease in the ATP yield per MJ glucose which occurs on the uncoupling of oxidative phosphorylation and the associated decrease in the ratio, amino acid : glucose energy (ATP/MJ amino acid $\div$ ATP/MJ glucose) were calculated as indicated in the Methods Section (p. 383). For each amino acid three curves are shown corresponding to uncoupling at site $1(-)$, sites $2+3(--)$ and uncoupling by proton leakage $(-)$. The physiologically relevant range for the whole body is probably between 10 and $13 \mathrm{ATP} / \mathrm{MJ}$ glucose (see p. 385). 


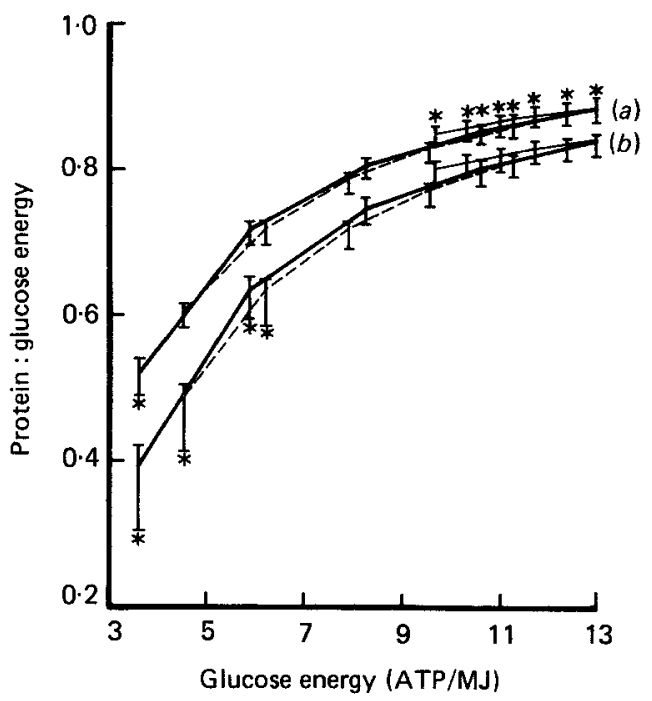

Fig. 3. Changes in the efficiency of oxidation of food proteins relative to that for glucose. The decrease in the ATP yield per MJ glucose which occurs on uncoupling of oxidative phosphorylation and the associated decrease in the ratio, protein: glucose energy (ATP/metabolizable MJ protein $\div$ ATP/MJ glucose) were calculated as indicated in the Methods Section (p. 383). Two sets of three curves are shown for oxidation of the protein directly $(a)$ and indirectly via gluconeogenesis $(b)$. Each set shows the effect of uncoupling at site $1(-)$, sites $2+3(---)$ and uncoupling by proton leakage $(-)$. The vertical bars show the range of values obtained for 101 food proteins whereas the curves drawn join points derived for lean beef protein. *Oxidative efficiencies when the metabolizable energy in gelatin is conserved as cytoplasmic ATP either more efficiently (above the vertical bar) or less efficiently (below the vertical bar) than the other 100 food proteins examined (see below). The physiologically relevant range for the whole body is probably between 10 and 13 ATP/MJ glucose (see p. 385).

conservation for the 101 food proteins is small (vertical bars, Fig. 3), an observation discussed in detail previously (Livesey, 1984) and in contrast to impressions given by others (Schulz, 1975; McGilvery, 1979). The vertical range bars (Fig. 3) generally increase, both absolutely (minimally fourfold) and relatively (minimally $44 \%$ of the mean value), with loss of energy conservation but remain nearly constant in the probable physiologically relevant range of energy coupling (i.e. between approximately 10 and $13 \mathrm{ATP} / \mathrm{MJ}$ glucose for these stoichiometries predicted on the basis that $>30 \%$ uncoupling would result in rapid fatigue as discusssed previously). A striking observation is that the energy in gelatin is conserved more efficiently than the energy present in any of the other food proteins when mitochondrial coupling is high and oxidation is direct, but is oxidized with least efficiency in severely uncoupled mitochondria (Fig. 3), whether directly oxidized or oxidized via glucose. As a food group, however, milk proteins are oxidized most efficiently under all conditions (see Livesey, 1984), including those when site 1 is selectively uncoupled (results not shown).

For medium- and long-chain fatty acids, uncoupling at site 1 raises the fatty acid energy value relative to that for glucose (Fig. 4). This contrasts with the effects on amino acids and of uncoupling by the other mechanisms. The pattern of effect for short-chain fatty acids is similar to that for the amino acids. Energy values were also obtained for unsaturated medium- and long-chain fatty acids but were little different from those shown for the corresponding carbon- 12 to carbon- 24 saturated fatty acids (Fig. 4), even when the number of $\mathrm{C}-\mathrm{C}$ double bonds accounted for extended to six (results not shown). A similar analysis showed that the glycerol:glucose energy value remains virtually constant except at very severe levels of uncoupling of oxidative phosphorylation, beyond that attainable by site 1 


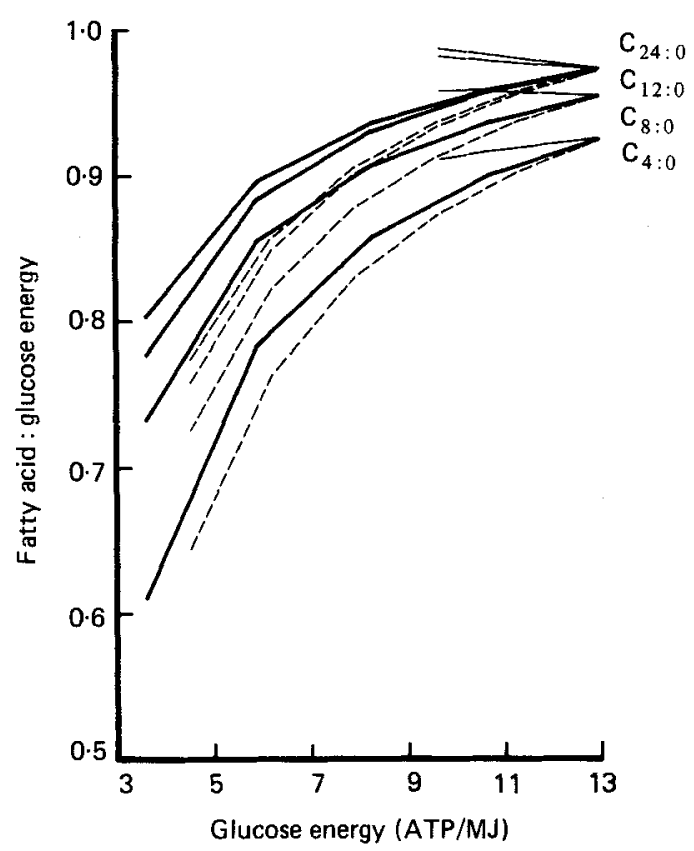

Fig. 4. Changes in the efficiency of oxidation of saturated fatty acids relative to that for glucose. The decrease in the ATP yield per MJ glucose which occurs on the uncoupling of oxidative phosphorylation and the associated decrease in the ratio, fatty acid: glucose energy (ATP/MJ fatty acid $\div$ ATP/MJ glucose) were calculated as indicated in the Methods Section (p. 383). Three curves are shown for each fatty acid corresponding to uncoupling at site 1 (- - ), sites $2+3(---)$ and uncoupling by proton leakage (-). The physiologically relevant range for the whole body is probably between 10 and 13 ATP/MJ glucose (see p. 385).

uncoupling alone (results not shown). The pattern of effects of uncoupling for fat or triglyceride will therefore resemble that for the medium- to long-chain fatty acids. Variation in the efficiency of energy conservation for those food fats with compositions given in food tables (Paul \& Southgate, 1978) is extremely small (Livesey, 1984) so that effects of site 1 uncoupling on this value were not investigated.

The large uncertainty in evaluating the efficiency of energy conservation during oxidation of energy-yielding nutrients (Livesey, 1984), the small variation in this efficiency when different food proteins are oxidized under any one metabolic condition and the change in rank order position of proteins for efficiency of energy conservation during protein oxidation with change in metabolic conditions (Fig. 3), compound to make a discrimination between the efficiency of energy conservation for one food protein and that for another of little practical value (with a range of $6 \%$ for a purely protein diet) and of no dietetic significance. Whereas site 1 uncoupling slightly decreases the energy value of protein relative to that for glucose (maximally $4 \%$ ), the energy value for fat increases (maximally $4 \%$ ). This differs from the effects of uncoupling by release of proton ejection from electron transfer at sites $2+3$ and uncoupling by proton leakage where both isodynamic equivalents decrease with decreasing efficiency of energy conservation (maximally $8 \%$ for protein and $5 \%$ for fat for the same extent of uncoupling). Nevertheless, the magnitude of the site 1 uncoupling effects are small and, for a mixed diet, would tend to cancel. The effects illustrated are predicted to be even smaller had proton stoichiometries ( $a, b$ and $c$ in Fig. 1) been used which yielded higher efficiencies of energy conservation in coupled mitochondria (and vice versa, 
see Livesey, 1984). The conclusion (Livesey, 1984) remains, therefore, that there is no compelling requirement for revision of the Atwater, or similar, factors to take account of differing efficiencies of protein, fat and carbohydrate oxidation. This conclusion seems to apply also to subjects with uncoupling at site 1 whether resulting from a mitochondrial myopathy (Clark et al. 1983) or induced by specific diets (Deaver et al. 1984). Except for the loss of energy conservation at coupling site 1 , this position still may be reversed if it could be shown that the efficiency of oxidative phosphorylation in vivo is much lower than presently considered.

The author is grateful to Mrs Susan M. Ring for computer programming and to Dr David A. T. Southgate for comments on the manuscript.

\section{REFERENCES}

Ball, E. G. (1973). Energy Metabolism. Boston: Addison Wesley.

Blaxter, K. L. (1967). The Energy Metabolism of Ruminants. London: Hutchinson Scientific Publications.

Chance, B. (1970). Proceedings of the National Academy of Sciences, USA 66, 1175-1182.

Clark, J. B., Hayes, D. J., Byrne, E. \& Morgan-Hughes, J. A. (1983). Biochemical Society Transactions 11, $626-627$.

Deaver, O. E., McCusker, R. H. \& Berdanier, C. D. (1984). Federation of American Societies for Experimental Biology 41, 745 (Abstract).

Hill, F. W. (1981). Federation Proceedings 30, 1434-1435.

Krebs, H. A. (1964). In Mammalian Protein Metabolism, vol. 1, pp. 125-176 [H. Munro and J. B. Allison, editors]. New York: Academic Press.

Livesey, G. (1984). British Journal of Nutrition 51, 15-28.

McGilvery, R. W. (1979). Biochemistry: a Functional Approach, 2nd ed. London: W. B. Saunders.

Merril, A. L. \& Watt, B. K. (1955) (revised 1973). Energy Values of Foods, Basis and Derivation. United States Department of Agriculture Handbook no. 74.

Miller, D. S. \& Judd, P. (1984). Journal of the Science of Food and Agriculture 35, 111-116.

Miller, D. S. \& Payne, P. R. (1959). British Journal of Nutrition 13, 501-508.

Morgan-Hughes, J. A. (1982). In Recent Advances in Clinical Neurology, pp. 1-46 [W. B. Mathews and G. H. Gazer, editors]. Edinburgh: Churchill Livingstone.

Morgan-Hughes, J. A., Landon, D. N., Land, J. M. \& Clark, J. B. (1979). Journal of Neurological Science 43, 27-46.

Nicholls, D. G. (1976). FEBS Letter 61, 103-110.

Nicholls, D. G. (1982). Bioenergetics: an Introduction to the Chemiosmotic Theory. New York: Academic Press.

Paul, A. A. \& Southgate, D. A. T. (1978). McCance \& Widdowson's The Composition of Foods, 4th ed. London: H.M. Stationery Office.

Rubner, N. (1902). In The Law of Energy Consumption in Nutrition, pp. 7-57 [R. T. Joy, editor]. Washington DC: US Army Research Institute.

Schulz, A. R. (1975). Journal of Nutrition 105, 200-207.

Skulachev, V. P. (1963). Proceedings of the 5th International Congress on Biochemistry, Moscow 5, 365-375. 